\title{
BMI
}

\section{Effect on smoking quit rate of telling patients their lung age: the Step2quit randomised controlled trial}

\author{
Gary Parkes, general practitioner,, ${ }^{1}$ Trisha Greenhalgh, professor , ${ }^{2}$ Mark Griffin, lecturer in medical \\ statistics, ${ }^{2}$ Richard Dent, consultant chest physician department of chest medicine ${ }^{3}$
}

\begin{abstract}
${ }^{1}$ The Limes Surgery, Hoddesdon, Hertfordshire EN11 8EP

${ }^{2}$ Department of Primary Care and Population Sciences, University College London, London N19 5LW

${ }^{3}$ Queen Elizabeth II Hospital, Welwyn Garden City, Hertfordshire AL7 4HQ

Correspondence to: $\mathrm{G}$ Parkes

Parkesko@hotmail.co.uk
\end{abstract}

doi:10.1136/bmj.39503.582396.25

\section{ABSTRACT}

Objective To evaluate the impact of telling patients their estimated spirometric lung age as an incentive to quit smoking.

Design Randomised controlled trial.

Setting Five general practices in Hertfordshire, England. Participants 561 current smokers aged over 35. Intervention All participants were offered spirometric assessment of lung function. Participants in intervention group received their results in terms of "lung age" (the age of the average healthy individual who would perform similar to them on spirometry). Those in the control group received a raw figure for forced expiratory volume at one second $\left(\mathrm{FEV}_{1}\right)$. Both groups were advised to quit and offered referral to local NHS smoking cessation services. Main outcome measures The primary outcome measure was verified cessation of smoking by salivary cotinine testing 12 months after recruitment. Secondary outcomes were reported changes in daily consumption of cigarettes and identification of new diagnoses of chronic obstructive lung disease.

Results Follow-up was $89 \%$. Independently verified quit rates at 12 months in the intervention and control groups, respectively, were $13.6 \%$ and $6.4 \%$ (difference $7.2 \%$, $\mathrm{P}=0.005,95 \%$ confidence interval $2.2 \%$ to $12.1 \%$; number needed to treat 14). People with worse spirometric lung age were no more likely to have quit than those with normal lung age in either group. Cost per successful quitter was estimated at $£ 280$ ( $€ 366, \$ 556)$. A new diagnosis of obstructive lung disease was made in $17 \%$ in the intervention group and $14 \%$ in the control group; a total of $16 \%$ (89/561) of participants.

Conclusion Telling smokers their lung age significantly improves the likelihood of them quitting smoking, but the mechanism by which this intervention achieves its effect is unclear.

Trial registration National Research Register N0096173751.

\section{INTRODUCTION}

A quarter of smokers develop chronic obstructive pulmonary disease (COPD), ${ }^{1}$ which is largely caused by smoking and is the fourth commonest cause of death worldwide. ${ }^{2}$ In the United Kingdom, half of the estimated 1.5 million people with chronic obstructive pulmonary disease are currently undiagnosed. According to the National Institute for Health and Clinical Excellence, the mean delay from onset to diagnosis is 20 years. ${ }^{4}$ Spirometry can detect obstructive lung damage in susceptible individuals after 20 pack years of smoking, typically at around age 35 . Yet the average age of diagnosis of chronic obstructive pulmonary disease in the UK is 55 , despite widespread availability of diagnostic equipment. ${ }^{4}$

Early diagnosis of chronic obstructive pulmonary disease with communication of lung damage to patients could improve targeting of smoking cessation programmes and improve quit rates in individuals most vulnerable to lung damage. ${ }^{5} \mathrm{~A}$ Cochrane review of the use of personal biomarkers (carbon monoxide measurements, spirometry, arterial damage) for the harmful effects of smoking, however, failed to find firm evidence that such markers could be used to increase the quit rate. ${ }^{6}$ A recent non-randomised observational study on the effect of communicating spirometry findings on smoking cessation concluded that "a large randomised clinical trial is needed to answer this important question more conclusively."

The concept of "lung age" (the age of the average person who has an $\mathrm{FEV}_{1}$ equal to the individual) was developed in 1985 as a way of making spirometry data easier to understand and also as a potential psychological tool to show smokers the apparent premature ageing of their lungs. ${ }^{5}$ We tested the hypothesis that telling smokers their lung age would lead to successful smoking cessation, especially in those with most damage.

\section{METHOD}

\section{Management and governance}

The research advisory group comprised a respiratory physician (RD), an academic general practitioner (TG), and the principal investigator (GP). A core management group, comprising principal investigator, practice manager, two practice nurses, healthcare assistant, and a patient representative, was responsible for the day to day running of the project.

\section{Sampling and recruitment}

A power calculation indicated the need for about 300 participants to have $80 \%$ power to detect a $10 \%$ 
Table 1 | Stages of change questions (adapted from Prochaska ${ }^{14}$ )

\begin{tabular}{lll}
$\begin{array}{l}\text { Stage } \\
\text { Pre-contemplative }\end{array}$ & Not even thinking about changing & \multicolumn{1}{c}{$\begin{array}{c}\text { Defining question } \\
\text { Answers "no" to the question "Are you intending to } \\
\text { quit smoking in the next } 6 \text { months?" }\end{array}$} \\
\hline Contemplative & Thinking about changing & $\begin{array}{l}\text { Answers "yes" to previous question and "no" to the } \\
\text { question "Are you intending to quit smoking in the } \\
\text { next month?" }\end{array}$ \\
\hline Preparation & Making plans to change & $\begin{array}{l}\text { Answers "yes" to previous two questions and "no" } \\
\text { to the question "Did you try to quit smoking in the } \\
\text { past year?" }\end{array}$ \\
\hline Action & Actively trying to change & $\begin{array}{l}\text { Answers "yes" to the question "Did you try to quit } \\
\text { smoking in the past year?" }\end{array}$ \\
\hline Maintenance* & Having achieved change, is trying to & $\begin{array}{l}\text { Answers "Yes"' to the question "Have you given up } \\
\text { smoking?" }\end{array}$ \\
\hline
\end{tabular}

*Question on "maintenance" phase used only in follow-up assessment as all participants were current smokers at baseline. assessment in 12 months. Baseline data included age, smoking history in pack years (average daily number of cigarettes smoked divided by 20 and multiplied by the number of years of smoking), medical history for exclusion criteria (see above), medication (especially use of steroids or antibiotics for chest infections in the preceding 12 months), and comorbidity including chronic bronchitis or emphysema, asthma, other lung disease, diabetes, treatment for blood pressure, stroke, coronary heart disease (angina or heart attack), or other heart disease. These comorbidities were not used as exclusion criteria but to confirm baseline comparability of groups.

All participants underwent standard measurements of lung function $\left(\mathrm{FEV}_{1}, \mathrm{FVC}\right.$ (forced vital capacity), $\mathrm{FEV}_{1} / \mathrm{FVC}$ ) with a Micromedical spirometer. Reversibility of airways obstruction was measured according to standard British Thoracic Society guidelines (over $15 \%$ and at least $400 \mathrm{ml}$ improvement in $\mathrm{FEV}_{1}$ after $400 \mu \mathrm{g}$ salbutamol via a spacer). ${ }^{8}$ Both groups were told that their lung function would be measured again after 12 months to see whether it had deteriorated. They were not randomised until after spirometry had been completed. All participants were strongly encouraged to give up smoking and advised how to access local NHS smoking cessation clinics.

We used two instruments to confirm baseline comparability of groups: the St George's respiratory questionnaire and Prochaska's stages of change questions in relation to smoking. The St George's respiratory questionnaire is a validated questionnaire designed to be self administered under supervision and to measure the impact of respiratory diseases (in particular asthma and chronic obstructive pulmonary disease) on an individual's life. ${ }^{9}$ Like other quality of life instruments, it has the potential to identify a threshold response to therapy or compare the response to different therapies, or both. ${ }^{10}$ Scores of 7 or below indicate normal lung function. We adapted stage of change questions (with permission) from Prochaska and DiClemente's model in which smokers are asked three questions and classified on the basis of their response as in the "pre-contemplative," "contemplative," "preparation," or "action"” phase (table 1).

\section{Randomisation procedure}

A clerk (who then took no further part in the study) prepared 600 sequentially numbered opaque sealed envelopes, each containing a card with allocation group determined by computer generated random number (odd = intervention). If the participant met the inclusion criteria and gave consent, he or she was entered into the study and underwent baseline spirometry. The next numbered envelope in the series was then opened to determine allocation group.

\section{Instruments and tests}

All data collectors were trained in the use of MicroLab 3500 spirometers (Micro Medical, Chatham, Kent), which were newly purchased at the start of the study. Spirometry readings were checked for internal
Fig 1 Graph of lung function against age showing how smoking accelerates age related decline in lung function (adapted from Fletcher and Peto ${ }^{11}$ ) 
reliability on three criteria: at least two $\mathrm{FEV}_{1}$ readings within $5 \%$ of each other; good quality time volume curve; and the internal spirometer computer display had to register "good blow." Smoking cessation at follow-up was initially assessed by measuring carbon monoxide concentrations with a Smoke Check SC01 monitor (Micro Medical, Chatham, Kent). This model has a carbon monoxide range of 0-500 ppm and a sensitivity of $1 \mathrm{ppm}$.

One of two independent nurses, who were blinded to allocation group, collected saliva samples for cotinine testing and recorded those who continued to take nicotine replacement therapy. Specimens were processed by ABS Laboratories, Medical Toxicology Unit, London. The optimum cut-off point to distinguish smokers from non-smokers is $14.2 \mathrm{ng} / \mathrm{ml}$, which correctly classifies $99 \%$ of non-smokers and $96 \%$ of smokers. As the half life of cotinine is about 20 hours, the test would detect most people who had smoked a cigarette within the past $24-48$ hours.

\section{Estimation of lung age}

Figure 1, adapted from the work of Fletcher and Peto, illustrates how smoking effectively "ages" the lungs. ${ }^{11}$ The examples illustrated show how the lungs can deteriorate more rapidly with smoking, as if they are ageing faster. Smoking cessation will not allow the lungs to return to normal but reduction in function or "ageing" will then occur at a normal rate. Originally, calculation of lung age was based on estimates developed by Morris and Temple with reference linear regression equations to establish the best method. They showed that $\mathrm{FEV}_{1}$ was the best test for calculating lung age mathematically (box). In practice, the lung age is automatically generated by adjustment of the settings of the spirometer.

\section{Information given to participants}

Participants in the intervention group were given their results verbally, immediately after randomisation, in the form of "lung age" with a graphic display (figs 1 and 2 ). The graphs were used as a visual aid to explain how the lung function normally reduces gradually with age and that smoking can damage lungs as if they are ageing more rapidly than normal. As an example a line can be draw vertically up from the horizontal axis (fig 2) from "age 52" to reach the bold blue curve illustrating the lung function of the "susceptible smoker" and then horizontally to the curve representing those who have "never smoked" and lung function at age 75 . Furthermore they were told that smoking cessation would slow

Lung age calculation formula developed by Morris and Temple

Men

Lung age $=2.87 \times$ height (in inches) $-\left(31.25 \times\right.$ observed $\mathrm{FEV}_{1}$ (litres) -39.375

Women

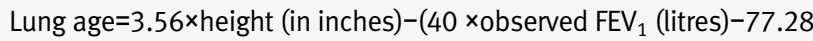

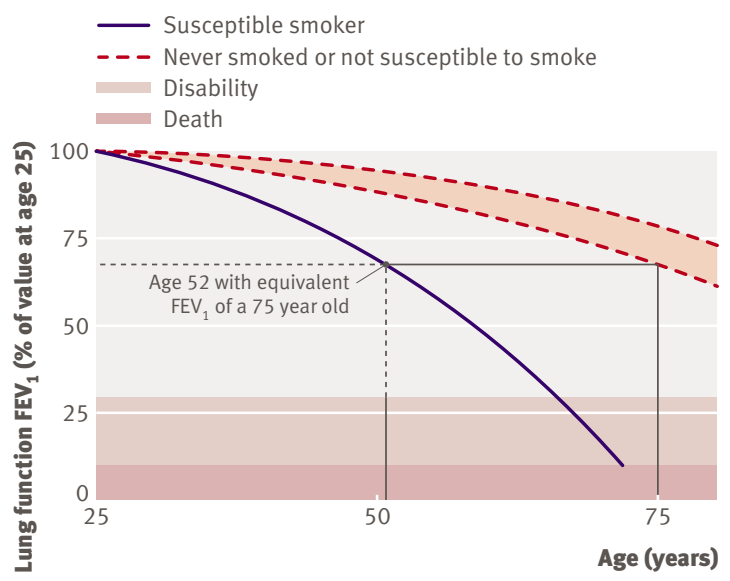

Fig 2 | Explaining lung age to participants (adapted from Fletcher and Peto ${ }^{11}$ )

down the rate of deterioration of the lung function back to normal but would not repair the damage already done.

In the intervention group, if the lung age was equal to or less than the individual's chronological age, he or she was informed that test result was normal. If lung age was greater than chronological age, we gave them the "lung age" in years.

We did not tell those in the control group their results but informed them that they would be invited for a second test after 12 months to "see if there had been any change in lung function." If the examiner was pressed for more information, he or she could tell participants that they would receive a letter with more information from the research doctor within four weeks.

The principal research doctor (GP) reviewed all the results, checked the quality of the spirometry tracing, and considered the result in the light of clinical data. When there was doubt, he sent the results to a chest physician $(\mathrm{RD})$ for interpretation and advice. Within four weeks of data collection the research doctor sent all participants an individualised letter. Written results were given to the control group as simple $\mathrm{FEV}_{1}$ (litres per second) with no further explanation. Written results were given to the intervention group as "lung age."

The letter to both groups included the phrase "This type of lung function test does not tell us anything about the risk of other serious diseases related to smoking such as lung cancer or heart disease or stroke. Smoking cessation is therefore still important for all people regardless of their age or the results of these lung tests." All participants were given written contact details of the local NHS smoking cessation services.

In both groups, when reversibility testing indicated asthma (over 15\% and at least $400 \mathrm{ml}$ improvement in $\mathrm{FEV}_{1}$ after $400 \mu \mathrm{g}$ salbutamol via a spacer) we advised participants to attend their general practitioner for further management, and informed the general practitioner separately. When spirometry findings suggested restrictive lung disease, we sent the participant and his or her general practitioner a letter to alert them to the 


\begin{tabular}{|c|c|c|}
\hline & Control $(n=281)$ & Intervention $(n=280)$ \\
\hline Age (years) & $53(11.9)$ & $52.9(11.9)$ \\
\hline$\%(\mathrm{No})$ of men & $47(132)$ & $45(127)$ \\
\hline Pack years & $30.3(19.3)$ & $31.1(17.7)$ \\
\hline Daily cigarette consumption & $17.4(8.2)$ & $16.5(9)$ \\
\hline \multicolumn{3}{|l|}{ Spirometry result: } \\
\hline $\mathrm{FEV}_{1} \%$ predicted & $90(19.8)$ & 89 (19.8) \\
\hline$\% \mathrm{FEV}_{1} / \mathrm{FVC}$ & $75(11.8)$ & $73(11.7)$ \\
\hline$\%$ with abnormal $\mathrm{FEV}_{1}$-that is, $180 \%$ of predicted $(\mathrm{No})$ & $23.5(66)$ & $26.8(75)$ \\
\hline SGRQ score & $28.9(22.4)$ & $26.7(22.0)$ \\
\hline \multicolumn{3}{|l|}{ \% (No) with medical history: } \\
\hline COPD & $7.2(19)$ & $7.7(20)$ \\
\hline Asthma & $11(29)$ & $9.3(24)$ \\
\hline Other lung disease & $2.7(7)$ & $2.3(6)$ \\
\hline CVA or stroke & $4.2(11)$ & $0.8(2)$ \\
\hline CHD (angina or heart attack) & $5.3(14)$ & $2.3(6)$ \\
\hline Other heart disease & $2.3(6)$ & $1.2(3)$ \\
\hline Diabetes & $5.7(15)$ & $3.5(9)$ \\
\hline Treatment for hypertension & $21.3(56)$ & $19.1(49)$ \\
\hline$\%$ (No) with new diagnosis of COPD & $17.4(49)$ & $14.3(40)$ \\
\hline \multicolumn{3}{|l|}{$\%(\mathrm{No})$ at stage of change: } \\
\hline No of patients* & 263 & 260 \\
\hline Pre-contemplative & $29.3(77)$ & $29.2(76)$ \\
\hline Contemplative & $32.3(85)$ & $31.9(83)$ \\
\hline Preparation & $16(42)$ & $18.1(47)$ \\
\hline Action & $22.4(59)$ & $20.8(54)$ \\
\hline
\end{tabular}

advisability of further investigation and guidelines on referral to secondary care.

\section{Outcome measures}

The primary outcome measure was verified cessation of smoking 12 months after the initial recruitment interview and examination. Secondary outcomes were changes in daily consumption of cigarettes and the identification of new diagnoses.

\section{Follow-up and confirmation of cessation}

Participants underwent follow-up examination with repeat spirometry after 12 months. Self reported quitters had carbon monoxide breath testing immediately for confirmation of smoking cessation, and they were informed that they would be contacted by an independent nurse for a saliva test for cotinine measurement.

\section{Data analysis}

We analysed data on an intention to treat basis and performed statistical analysis with SPSS version 11.0. We used unpaired $t$ tests for continuous data and $\chi^{2}$ tests for categorical data, except when expected cells were found to be less than 5, in which case we used Fisher's exact test.

To test the hypothesis that severity of lung damage predicts quit success, we used the $t$ test to compare the mean "lung age deficit" (difference of lung age minus chronological age) between quitters and non-quitters within the intervention group.

\section{Assessment of costs}

Though we did not carry out a full economic evaluation, we had accurate data on the time taken to carry out the spirometry tests and for results to be communicated to patients by letter. We calculated costs in terms of the time spent per patient processed and also per successful quitter.

\section{RESULTS}

Baseline characteristics

We recruited 561 participants (table 2). There were few significant differences between the groups at baseline, in particular groups did not differ in their quality of life score or stage of change. There were, however, significantly more people with a history of stroke in the control group. The incidence of comorbidity was high (around 20\% of all participants), reflecting our deliberate intention not to exclude high risk individuals (and, perhaps, the inability or unwillingness of many smokers to quit despite the presence of considerable medical morbidity).

Despite an average of 33 pack years of smoking, most participants in this study had "normal" results on spirometry at baseline, which accords with previous studies on comparable populations. ${ }^{1}$ According to British Thoracic Society cut-off values, only $23.5 \%$ of the control group and 26.8\% of the intervention group had baseline lung function in the "abnormal" range.

\section{Progress and outcome}

Figure 3 shows progress through the trial and losses to follow-up. Table 3 shows follow-up data at 12 months. All recruited participants were included in the final data analysis. We analysed those who did not return for follow-up (32 and 31, respectively, in the control and intervention group) as if they continued to smoke. Verified quit rates were $6.4 \%(18 / 281)$ in the control group and $13.6 \%(38 / 280)$ in the intervention group (difference $7.2 \%, \mathrm{P}=0.005,95 \%$ confidence interval $2.2 \%$ to $12.1 \%$ ). Telling participants their lung age was thus associated with an absolute reduction of $7.2 \%$ in the smoking rate compared with giving them their lung function tests results as raw $\mathrm{FEV}_{1}$ data. The number needed to treat (NNT) for the intervention to achieve one additional sustained quitter is 14 . Both groups reduced their average self reported consumption of cigarettes (table 3); average consumption at follow-up was significantly lower in the intervention group than in the control group (11.7 (SD 9.7) $v 13.7$ (SD 10.5) per day, $\mathrm{P}=0.03)$.

We recorded the numbers of smokers in both groups who used additional help to quit (health service clinics, nicotine replacement, bupropion, acupuncture, hyp nosis): numbers were $22(7.8 \%)$ in the control group and $30(10.7 \%)$ in the intervention group $(\mathrm{P}=0.2)$.

Within the intervention group we investigated the effect of lung age deficit (lung age minus actual age) on 
the likelihood of quitting. To investigate whether those with poorer lung function were more likely to quit we used independent samples $t$ test to compare the mean deficit between those who were confirmed to have stopped smoking $(n=38)$ versus the rest $(n=242)$. The mean lung age deficit was 8.7 years and 9.4 years in the quitters and non-quitters, respectively. This difference was not significant (difference in means $-0.78,95 \%$ confidence interval -7.6 to $6.0, \mathrm{P}=0.8$ ). Thus, there was no evidence that those individuals with poorer lung age deficits were more likely to quit. The study was not powered to investigate this relation, however, and the lack of a significant result might be because of the small numbers, particularly in the quit group.

\section{Costs}

It took a healthcare assistant 30 minutes to perform a spirometry test. The principal investigator (GP) spent a further 15 minutes per patient reviewing results and preparing an individualised feedback letter, and this required about 10 minutes of secretarial and receptionist support. Using 2007 salary costs for the relevant staff, we estimate the cost of this intervention at $£ 20$ $(€ 26, \$ 40)$ per patient processed and $£ 280(€ 366, \$ 556)$ per successful quitter (given a number needed to treat of 14).

\section{DISCUSSION}

This large randomised controlled trial with adequate follow-up and independent proof of cessation has shown that individualised feedback of "lung age" is effective in promoting smoking cessation. This study strongly supports the policy of giving patients their spirometry results expressed as "lung age" along with advice about the dangers of continuing to smoke and methods of quitting.

\section{Comparison with other research}

In 2001 a non-systematic overview analysed 12 studies that provided feedback on personal biomarkers as part of strategies to change behaviour in smokers. ${ }^{12}$ The authors concluded that success was likely to depend on how the information was conveyed and understood and how it related to behaviour. They also suggested that success might depend on graphic displays or

\begin{tabular}{|c|c|c|c|}
\hline & Control $(n=281)$ & Intervention $(\mathrm{n}=\mathbf{2 8 0})$ & $P$ value \\
\hline Lost to follow-up & $11.4(32)$ & $11.0(31)$ & 0.9 \\
\hline \multicolumn{4}{|l|}{ Smoking status } \\
\hline Confirmed cessation* & $6.4(18)$ & $13.6(38)$ & 0.01 \\
\hline Smoker at 12 months & $90.4(254)$ & $84.6(237)$ & \\
\hline Unknown & $3.2(9)$ & $1.8(5)$ & \\
\hline Mean (SD) daily cigarette consumption & $13.7(10.5)$ & $11.7(9.7)$ & 0.03 \\
\hline Attended NHS smoking clinics & $1.4(4)$ & $1.7(5)$ & \\
\hline $\begin{array}{l}\text { Used smoking cessation help (clinic, NRT, } \\
\text { bupropion, acupuncture) }\end{array}$ & $7.8(22)$ & $10.7(30)$ & $0.2 \dagger$ \\
\hline $\begin{array}{l}\text { NRT=nicotine replacement therapy. } \\
{ }^{*} \text { Cotinine and CO measurement. } \\
t^{2} \text { test. }\end{array}$ & & & \\
\hline
\end{tabular}

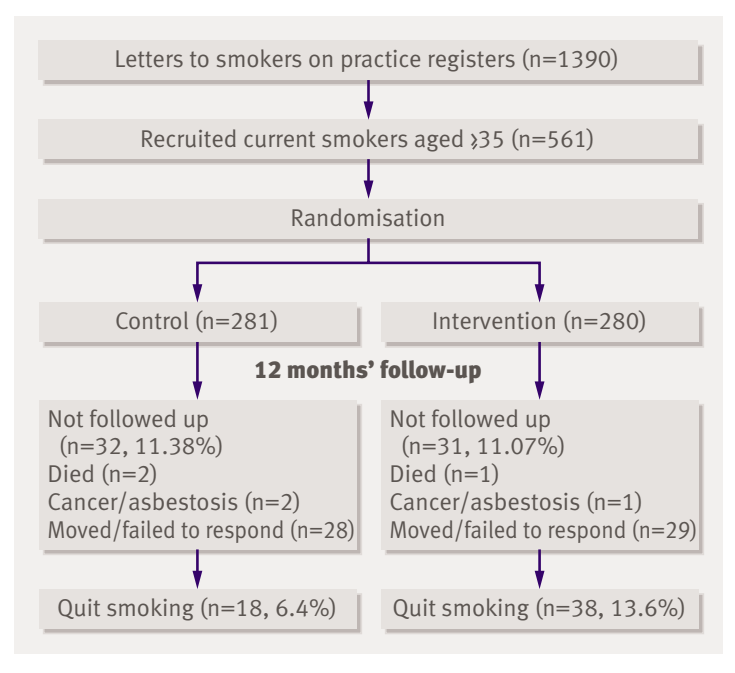

Fig 3 | Flow of participants through trial

written individualised information as well as the prospect of gain rather than negative messages about costs or disadvantage.

A Cochrane review of the evidence for the effectiveness of biomarkers in smoking cessation was published in October 2005. ${ }^{6}$ Observational studies were included in the background discussion but only randomised controlled trials were included in the analysis, which concluded that because of limited evidence no definitive statements could be made about the effectiveness of assessment of biomarkers as an aid for smoking cessation. ${ }^{6}$ None of the primary studies included in the Cochrane review had used "lung age" in the intervention. The negative conclusions of that review should be updated in the light of this new study.

The debate about the usefulness of screening with spirometry was recently rekindled by a large nonrandomised observational study of 4494 smokers from Poland. ${ }^{7}$ Their results indicated that spirometry promoted cessation. Those with airways obstruction were more likely to quit, but even the group with normal lungs on spirometry had a higher quit rate $(12 \%)$ than would normally be expected after simple advice from a physician (4-6\%). ${ }^{13}$ They did not use "lung age" to explain results to participants but did use a visual display of Fletcher and Peto's diagram ${ }^{11}$ to compare the participant's result with the average for age and project the likely deterioration with continued smoking. These authors did not have a control group but attributed the high quit rates in those with normal lung function to a "healthy volunteer" effect (those who had opted for the programme were seen as more motivated to quit).

The results of our study are broadly consistent with the findings of the Polish observational study, with one important difference. Contrary to the conclusions of the latter (and to clinical speculation), we found no evidence that successful quitting depends on the severity of lung damage as demonstrated by spirometry. Our study, however, was not powered to detect this difference, and we found, for example, that a 45 year 


\section{WHAT IS ALREADY KNOWN ON THIS TOPIC}

There is insufficient evidence to make a definitive statement about the evidence for the effectiveness of biomarkers (including spirometry) in smoking cessation

\section{WHAT THIS STUDY ADDS}

Smoking cessation rates can be improved by reporting estimation of lung age with spirometry in primary care

Screening smokers over the age of 35 could reduce smoking and improve early diagnosis of chronic obstructive pulmonary disease

old smoker who is told that their "lung age" is normal is as likely to quit as one who is told that his or her "lung age" is 65. Presentation of information in an understandable and visual way, whether the news is positive or negative, seems to encourage higher levels of successful smoking cessation than when patients are given feedback that is not easily understandable.

\section{What makes people quit}

What triggers the decision to quit and which methods result in successful and sustained quitting? Clinical experience suggests that deterioration in health does not necessarily lead to altered behaviour, whether that is related to smoking, drugs, or diet. The high rate of comorbidity $(20 \%)$ in our participants confirms that many people who are likely to exacerbate a chronic health problem by smoking continue to smoke. Anecdotally, some participants in our trial were relieved when the results were found to be normal and therefore thought it was "not too late" to be trying to quit.

This apparent win-win situation might explain the apparently paradoxical finding that knowing one's lung age helps a smoker to quit whatever the result. If lung age is normal there is an incentive to stop before it is too late. If lung age is abnormal then this is a clear message that the lungs are undergoing accelerated deterioration that would be slowed if the smoker stopped. Further research is needed to elucidate the psychological forces that are active in successful quitting in different circumstances.

In this study, we measured stage of change (using Prochaska and DiClemente's transtheoretical model ${ }^{14}$ ) to ensure that the groups were comparable for this variable at baseline, but the study was underpowered to test the hypothesis that a smoker in the "active" phase of quitting would find feedback on lung age more useful than someone in the "pre-contemplative" phase. Some addiction experts have proposed that the transtheoretical model should be rejected in favour of a new integrated model..$^{15}$ Any new psychological theory of smoking cessation will need to explain the unexpected finding that normal results within personal biomarkers are as likely to promote cessation as abnormal ones.

Current National Institute for Health and Clinical Excellence guidelines include one on brief interventions and referral for smoking cessation ${ }^{17}{ }^{18}$ (which do not mention spirometry testing at all) and another on the management of chronic obstructive pulmonary disease. ${ }^{4}$ The implication is that spirometry testing is useful only when the patient has (or is suspected of having) established lung damage. Our results suggest that both these guidelines should be reviewed and that lung age testing (which is a quick, office based test that can be undertaken by a healthcare assistant) should be considered as part of a brief intervention packageeither in all smokers over 35 (the lower age limit for this study) or all smokers. Currently the new contract for general practitioners in the UK includes incentives to confirm the diagnosis of chronic obstructive pulmonary disease with spirometry and to record smoking status in those with a record of relevant comorbidity (coronary heart disease, hypertension, diabetes, stroke, and asthma) and to give cessation advice. There is no incentive, however, to actively find cases of chronic obstructive pulmonary disease among smokers (or exsmokers) in these high risk groups or in the general population. We recommend that the new UK NHS general practitioner contract should include incentives for spirometric assessment accompanied by individualised communication of lung age in smokers.

Our cost estimates, which assume that spirometry is carried out in UK general practice, suggest that estimation and communication of lung age is of comparable effectiveness to, and potentially cheaper than, other currently available treatments on the NHS, including nicotine replacement therapy, ${ }^{19}$ bupropion, ${ }^{20}$ face to face counselling, ${ }^{21}$ and telephone counselling. ${ }^{22}$ Given the heavy health and economic burden of smoking, we believe that formal economic evaluation of this new and simple intervention should be a research priority.

We thank Liane Andrews (healthcare assistant) and members of the practice based steering group of nurses, manager, and lay members. We also thank the doctors and staff of the five local general practice surgeries for their help and cooperation. We thank P W Jones for permission to use the St George's respiratory questionnaire and for supplying copies of the questionnaire and the electronic scoring tool.

Contributors: All authors made a substantial contribution to: conception and design, or analysis and interpretation of data, drafting the article or revising it critically for important intellectual content, and final approval of the version to be published. GP conceived, designed, and piloted the original study. The work formed part of a research PhD thesis at University College London, with TG and RD as supervisors. MG supplied statistical advice and analysis and wrote the statistical section. GP is guarantor. Funding: Leading practice through research award from the Health Foundation.

Competing interests: None declared

Ethical approval: Hertfordshire local research ethics committee (application number EC03718) and West Essex local research ethics committee (1608-0104).

Provenance and peer review: Not commissioned; externally peer reviewed.

1 Van Schayck CP, Loozen JM, Wagena E, Akkermans RP, Wesseling G. Detecting patients at a high risk of developing chronic obstructive pulmonary disease in general practice: cross sectional case finding study. BMJ 2002;324:1370.

2 Price D, Duerden M. Chronic obstructive pulmonary disease. BMJ 2003;326:1046-7.

3 Soriano JB, Maier WC, Egger P, Visick G, Thakrar B, Sykes J, et al. Recent trends in physician diagnosed COPD in women and men in the UK. Thorax 2000;55:789-94.

4 National Collaborating Centre for Chronic Conditions. Chronic obstructive pulmonary disease. National clinical guideline on management of chronic obstructive pulmonary disease in adults in primary and secondary care. Thorax 2004;59(suppl 1):1-232 
5 Morris JF, Temple W. Spirometric "lung age" estimation for motivating smoking cessation. Prev Med 1985;14:655-62.

6 Bize R, Burnand B, MuellerY, Cornuz J. Biomedical risk assessment as an aid for smoking cessation. Cochrane Database Syst Rev 2005;(4):CD004705.

7 Bednarek M, Gorecka D, Wielgomas J, Czajkowska-Malinowska M, Regula J, Mieszko-Filipczyk G, et al. Smokers with airway obstruction are more likely to quit smoking. Thorax 2006;61:869-73.

8 British Thoracic Society. Clinical information. COPD. www.britthoracic.org.uk/Clinicallnformation/tabid/66/Default.aspx.

9 Jones PW, Quirk FH, Baveystock CM. The St George's respiratory questionnaire. Respir Med 1991;85(suppl B):25-31.

10 Jones PW, Quirk FH, Baveystock CM. Why quality of life measures should be used in the treatment of patients with respiratory illness. Monaldi Arch Chest Dis 1994;49:79-82.

11 Fletcher C, Peto R. The natural history of chronic airflow obstruction. $B M J$ 1977;i:1645-8.

12 McClure JB. Are biomarkers a useful aid in smoking cessation? A review and analysis of the literature. Behav Med 2001;27:37-47.

13 Lancaster T, Stead LF. Physician advice for smoking cessation. Cochrane Database Syst Rev 2004;(4):CD000165
14 Prochaska JO, DiClemente CC. Stages and processes of self-change of smoking: toward an integrative model of change. J Consult Clin Psychol 1983;51:390-5.

15 West R. Theory of addiction. 1st ed. Oxford: Blackwell, 2006.

16 West R, West R. Time for a change: putting the transtheoretical (stages of change) model to rest. Addiction 2005;100:1036-9.

17 National Institute for Health and Clinical Excellence. Smoking cessation: draft guidance. NICE,

2003. http://guidance.nice.org.uk/page.aspx?o=429278.

18 National Institute for Health and Clinical Excellence. Brief interventions and referral for smoking cessation in primary care and other settings. NICE, 2006. www.nice.org.uk/PHI001.

19 Silagy C, Lancaster T, Stead L, Mant D, Fowler G. Nicotine replacement therapy for smoking cessation. Cochrane Database Syst Rev 2004;(3):CD000146

20 Hughes JR, Stead LF, Lancaster T. Antidepressants for smoking cessation. Cochrane Database Syst Rev 2004;(4):CD000031.

21 Lancaster T, Stead LF. Individual behavioural counselling for smoking cessation. Cochrane Database Syst Rev 2005;(2):CD001292.

22 Stead LF, Lancaster T, Perera R. Telephone counselling for smoking cessation. Cochrane Database Syst Rev 2003;(1):CD002850.

Accepted: 30 January 2008 